\title{
Respuesta al comentario «Prevalencia de enfermedades de transmisión sexual en pacientes con VIH/SIDA»
}

\section{Reply to comment "Prevalence of sexually transmitted diseases in HIVIAIDS patients"}

\author{
Carmen G. Santamaría-Burgos*, Carlos E. Oliva-Torres, Catherin R. Molina-Valencia, \\ Jefferson X. Molina-Quimbita y Melanie M. Noriega-Moreno \\ Unidad de Atención Integral de personas viviendo con VIH/SIDA, Hospital General Enrique Garcés, Quito, Ecuador
}

Muy agradecidos por las observaciones realizadas a nuestro artículo científico titulado «Prevalencia de enfermedades de transmisión sexual en pacientes con $\mathrm{VIH} / \mathrm{SIDA}$ ", adjuntamos las respuestas a las observaciones realizadas.

Con respecto a la generalización del título y las enfermedades de transmisión sexual (ETS) encontradas, detallamos que considerando la prevalencia de las ETS del artículo científico evidenciamos que prepondera la sífilis en pacientes con identidad sexual homosexual e infección por el virus de la inmunodeficiencia humana $(\mathrm{VIH})$; sin embargo, se detalla además que existen limitantes en los datos, como una baja prevalencia de ETS y su distribución en el sexo femenino, para encontrar relaciones y generalizaciones significativas.

Con respecto a la predominancia de la sífilis en los pacientes con VIH, los estudios ${ }^{1,2}$ con los cuales se realiza la discusión verifican que existe una mayor prevalencia de sífilis. Se detalla que los hombres que tienen sexo con hombres presentan una mayor cantidad de ETS debido, con toda probabilidad, a que la práctica de la relación sexual anogenital representa un mayor riesgo de contagio; para la infección genital en mujeres no se realiza un estudio comparativo por su baja prevalencia. En la recolección de datos no se logra identificar infecciones por el virus de la hepatitis $C$, por lo cual no se realiza una correlación estadística y con artículos relacionados.

En la investigación se analizaron todas las historias clínicas de los 1186 pacientes infectados con $\mathrm{VIH}$, de los cuales 164 tuvieron ETS, evidenciando sífilis, verrugas anogenitales, candidiasis genital, herpes genital, donovanosis y gonorrea, por lo que el análisis comparativo se realiza con estas infecciones.

\section{Financiamiento}

La presente investigación no ha recibido ayudas específicas provenientes de instituciones del sector público, sector comercial o entidades sin ánimo de lucro.

\section{Conflicto de intereses}

Los autores declaran no tener ningún conflicto de intereses.

\section{Correspondencia:}

${ }^{*}$ Carmen G. Santamaría-Burgos

E-mail: c_santamaria2000@yahoo.es 0210-5187/@ 2021 Colegio Ibero Latinoamericano de Dermatología A.C. (CILAD). Publicado por Permanyer. Este es un artículo open access bajo la licencia CC BY-NC-ND (http://creativecommons.org/licenses/by-nc-nd/4.0/).

Disponible en internet: 28-01-2022 Med Cutan Iber Lat Am. 2022;50(1):56-57 www.MedicinaCutanealLA.com 


\section{Responsabilidades éticas}

Protección de personas y animales. Los autores declaran que para esta investigación no se han realizado experimentos en seres humanos ni en animales.

Confidencialidad de los datos. Los autores declaran que han seguido los protocolos de su centro de trabajo sobre la publicación de datos de pacientes.

Derecho a la privacidad y consentimiento informado. Los autores han obtenido el consentimiento informado de los pacientes y/o sujetos referidos en el artículo. Este documento obra en poder del autor de correspondencia.

\section{Bibliografía}

1. Mayer K. Which HIV-infected men who have sex with men in care are engaging in risky sex and acquiring sexually transmitted infections: findings from a Boston community health centre. Sex Transm Infect. 2010;86:66.

2. Motta A, Marín D, Merlo E. Prevalencia de herpes, sífilis y condilomatosis en pacientes con VIH en un hospital de referencia de Bogotá. Rev Asoc Colomb Dermatol. 2013;21:325 